\title{
Interfacial forces and Marangoni flow on a nematic drop retracting in an isotropic fluid
}

\author{
Pengtao Yue ${ }^{a}$, James J. Feng ${ }^{\mathrm{a}, *}$, Chun Liu ${ }^{\mathrm{b}}$, Jie Shen ${ }^{\mathrm{c}}$ \\ a Department of Chemical and Biological Engineering and Department of Mathematics, University of British Columbia, Vancouver, BC V6T 1Z4, Canada \\ ${ }^{\mathrm{b}}$ Department of Mathematics, The Pennsylvania State University, University Park, PA 16802, USA \\ ${ }^{c}$ Department of Mathematics, Purdue University, West Lafayette, IN 47907, USA
}

Received 30 December 2004; accepted 3 April 2005

Available online 27 April 2005

\begin{abstract}
Nematic-isotropic interfaces exhibit novel dynamics due to anchoring of the liquid crystal molecules on the interface. The objective of this study is to demonstrate the consequences of such dynamics in the flow field created by an elongated nematic drop retracting in an isotropic matrix. This is accomplished by two-dimensional flow simulations using a diffuse-interface model. By exploring the coupling among bulk liquid crystal orientation, surface anchoring and the flow field, we show that the anchoring energy plays a fundamental role in the interfacial dynamics of nematic liquids. In particular, it gives rise to a dynamic interfacial tension that depends on the bulk orientation. Tangential gradient of the interfacial tension drives a Marangoni flow near the nematic-isotropic interface. Besides, the anchoring energy produces an additional normal force on the interface that, together with the interfacial tension, determines the movement of the interface. Consequently, a nematic drop with planar anchoring retracts more slowly than a Newtonian drop, while one with homeotropic anchoring retracts faster than a Newtonian drop. The numerical results are consistent with prior theories for interfacial rheology and experimental observations.
\end{abstract}

(C) 2005 Elsevier Inc. All rights reserved.

Keywords: Dynamic interfacial tension; Liquid crystals; Anchoring energy; Two-phase flow

\section{Introduction}

Nematic-isotropic interfaces occur in a variety of composite materials, of which liquid-crystalline polymer (LCP) blends and polymer-dispersed liquid crystals (PDLC) are well-known examples [1,2]. An LCP blend consists of LCP droplets dispersed in an immiscible thermoplastic matrix. Under certain flow conditions, the LCP droplets may be stretched into thin, continuous threads which, owing to the nematic liquid-crystalline nature of the LCP, possess a high degree of molecular alignment in the axial direction. Upon solidification, these threads become fibers with exceptional strength and modulus and will act as in situ reinforcement for the composite material. Thus, polymer blends with an

\footnotetext{
* Corresponding author.

E-mail address: jfeng@chml.ubc.ca (J.J. Feng).
}

LCP minor phase are "self-reinforcing," and are also known as in situ composites [3,4]. PDLC materials are made of micrometer-size droplets of a small-molecule liquid crystal embedded in a polymer binder. The optical response of the droplets combined with the mechanical properties of the polymer make PDLC films a unique component in many electro-optical applications [2,5].

These technological advances require an understanding of the physics of nematic-isotropic interfaces. Perhaps the most fundamental issue is the interfacial tension. Continuum models and mean-field and atomistic calculations have predicted the easy axis on the interface and the magnitude of the interfacial energy [6-10]. A somewhat more advanced issue is the interfacial rheology, i.e., the dynamic coupling between the interfacial configuration and a flow field. In a series of papers, Rey [11-14] has presented a theoretical framework for the rheology and fluid dynamics of nematicisotropic interfaces. Three notable features are: (a) The inter- 
facial tension may be orientation-dependent; (b) tangential gradients of the interfacial tension give rise to Marangoni flows from regions of low anchoring energy toward regions of high anchoring energy; and (c) the surface stress tensor has an anisotropic elastic contribution that produces a bending stress when the orientation deviates from the easy axis.

Intriguing as Rey's theory is, so far it has not been used in any flow calculation. This is perhaps understandable since tracking a moving interface is a difficult numerical task by itself, and this difficulty is compounded here by the peculiar rheology of the nematic fluid. The only attempt at a dynamic calculation appears to be that of Popa-Nita and Sluckin [15], who used a diffuse-interface model to circumvent the difficulty of tracking the interface, and made drastic simplification about the flow-orientation coupling at the interface. Specifically, the director orientation is assumed to be fixed in space and time, uncoupled from the flow; the changes in the liquid-crystalline conformation are represented solely by a scalar order parameter. Furthermore, the nematic-isotropic interface remains at the transition temperature with zero interfacial energy. This simplified picture may have captured the essential physics for their particular problem, namely the propagation and dispersion of a small-amplitude wave. But it is obviously inadequate for stronger deformation of a general nematic-isotropic two-phase system as encountered, say, in the processing of LCP blends or PDLC films.

This paper represents our effort toward dynamic simulation of nematic-isotropic two-phase flows. We have previously developed a diffuse-interface formalism for two-phase flows of general complex fluids [16], and will apply the methodology to simulate an elongated nematic drop that retracts in a quiescent isotropic Newtonian fluid. Our rationale in choosing this problem is two-fold. First, it is a relatively simple flow situation that clearly illustrates the dynamic coupling among the three major factors: the nematic orientation inside the drop, the surface anchoring, and the flow field in both components. The simulation is based on a theory that is essentially the diffuse-interface analogue of Rey's theory [13]. The diffuse interface being a numerical device (cf. Section 2), ours will be the first fluid mechanical simulations incorporating the proper interfacial rheology. Not surprisingly, they reproduce the three effects listed above that are anticipated by Rey $[12,13]$. Second, this problem is relevant to using drop retraction as a means to measure interfacial tension between the two components $[17,18]$. Thus, there are experimental observations $[19,20]$ with which our results can be compared. We will confine our discussion to nematic drops in a Newtonian matrix. The retraction of Newtonian drops in a nematic matrix has been described elsewhere [21].

In the next section, we will describe the theoretical model and the numerical method. Section 3 will analyze the numerical results in detail and compare them to prior work. Section 4 will summarize the whole paper and point out the limitations of our work.

\section{Theoretical model and numerical method}

The difficulty of moving interface problems stems from the fact that the interfacial movement is naturally amenable to a Lagrangian description while the fluid flow is conventionally solved in an Eulerian framework. Aside from this mathematical dilemma, the flow of multi-component complex fluids also involves the physical problem of microstructure-dependent rheology. Recently, we proposed a diffuseinterface model for two-phase flows of complex fluids that resolves both issues at once [16]. In this model, the two components are assumed to mix in a narrow interfacial layer, across which physical properties change steeply but continuously. The interfacial position and thickness are determined by a phase-field variable whose evolution is governed by a mixing energy. This way, the structure of the interface is rooted in molecular forces and calculated from a convection-diffusion equation; there is no longer a need for tracking the interface. Another advantage of our model, critical to the task at hand, is its capability of easily incorporating the rheology of microstructured fluids. As long as the conformation of the microstructure is describable by a free energy, this energy can be added to the mixing energy to form the total free energy of the multi-phase system. Then a formal variational procedure applied to the total free energy will give rise to the proper constitutive equation for the microstructured fluids in addition to the evolution equation of the phase-field variable. Dissipative effects such as viscous stresses, of course, have to be accounted for separately, e.g., via the standard irreversible thermodynamic procedure [22]. Our treatment of the interface resembles that of Popa-Nita and Sluckin [15]. But we allow fully dynamic coupling between the interface, the nematic orientation and the fluid flow, and thus are able to carry out fluid dynamical simulations based on continuum-level governing equations.

Yue et al. [16] have given a detailed derivation of the model, described its numerical implementation using a spectral method and presented preliminary results for validation. We will only summarize the salient features here.

Consider an "immiscible" blend of a Newtonian liquid and a nematic liquid crystal. The diffuse-interface has a small but nonzero thickness, inside which the two components are mixed and store a mixing energy. We define a phase-field variable $\phi$ such that the concentrations of the nematic and Newtonian components are $(1+\phi) / 2$ and $(1-\phi) / 2$, respectively. Then $\phi=1$ in the bulk nematic phase, and $\phi=-1$ in the bulk Newtonian phase. The interface is taken to be the level set $\phi=0$. There are three types of free energies in this system: mixing energy of the interface, bulk distortion energy of the nematic, and the anchoring energy of the liquid crystal molecules on the interface. For these we adopt the following forms:

$$
\begin{aligned}
& f_{\text {mix }}=\frac{\lambda}{2}|\nabla \phi|^{2}+\frac{\lambda}{4 \epsilon^{2}}\left(\phi^{2}-1\right)^{2}, \\
& f_{\text {bulk }}=K\left[\frac{1}{2} \nabla \boldsymbol{n}:(\nabla \boldsymbol{n})^{\mathrm{T}}+\frac{\left(|\boldsymbol{n}|^{2}-1\right)^{2}}{4 \delta^{2}}\right],
\end{aligned}
$$


$f_{\text {anch }}=\left\{\begin{array}{l}\frac{A}{2}(\boldsymbol{n} \cdot \nabla \phi)^{2} \quad \text { for planar anchoring, } \\ \frac{A}{2}\left[|\boldsymbol{n}|^{2}|\nabla \phi|^{2}-(\boldsymbol{n} \cdot \nabla \phi)^{2}\right] \\ \quad \text { for homeotropic anchoring. }\end{array}\right.$

In $f_{\text {mix }}, \lambda$ is the mixing energy density, $\epsilon$ is the capillary width and the ratio $\lambda / \epsilon$ produces the interfacial tension $\sigma$ $[23,24] . f_{\text {bulk }}$ is the Frank energy with a single elastic constant $K, \boldsymbol{n}$ being the director, regularized to permit defects where $|\boldsymbol{n}|$ deviates from unity over a small region of size $\delta$ $[25,26]$. For $f_{\text {anch }}$, we adapt the Rapini-Popoular form [6] to our diffuse-interface formalism, with $A$ being the anchoring energy density. Now we have the total free energy density for the two-phase material:

$f(\phi, \boldsymbol{n}, \nabla \phi, \nabla \boldsymbol{n})=f_{\text {mix }}+\frac{1+\phi}{2} f_{\text {bulk }}+f_{\text {anch }}$.

Following a variational procedure detailed in Ref. [16], one may derive the evolution equations for the configuration variables $\boldsymbol{v}, \phi$ and $\boldsymbol{n}$. Supplemented by the appropriate dissipative terms, these are essentially the governing equations familiar in the classical context,

$\nabla \cdot \boldsymbol{v}=0$

$\rho\left(\frac{\partial \boldsymbol{v}}{\partial t}+\boldsymbol{v} \cdot \nabla \boldsymbol{v}\right)=-\nabla p+\nabla \cdot \boldsymbol{\sigma}$,

$\frac{\partial \phi}{\partial t}+\boldsymbol{v} \cdot \nabla \phi=\gamma_{1} \lambda \nabla^{2}\left[-\nabla^{2} \phi+\frac{\phi\left(\phi^{2}-1\right)}{\epsilon^{2}}\right]$,

$\frac{\partial \boldsymbol{n}}{\partial t}+\boldsymbol{v} \cdot \nabla \boldsymbol{n}=\gamma_{2} \boldsymbol{h}$,

where the deviatoric stress tensor $\sigma$ is

$$
\begin{aligned}
\boldsymbol{\sigma}= & -\lambda(\nabla \phi \otimes \nabla \phi)-K \frac{1+\phi}{2}(\nabla \boldsymbol{n}) \cdot(\nabla \boldsymbol{n})^{\mathrm{T}} \\
& -\boldsymbol{G}+\mu\left[\nabla \boldsymbol{v}+\left(\nabla \boldsymbol{v}^{\mathrm{T}}\right)\right],
\end{aligned}
$$

with $\boldsymbol{G}=A(\boldsymbol{n} \cdot \nabla \phi) \boldsymbol{n} \otimes \nabla \phi$ for planar anchoring and $\boldsymbol{G}=$ $A[(\boldsymbol{n} \cdot \boldsymbol{n}) \nabla \phi-(\boldsymbol{n} \cdot \nabla \phi) \boldsymbol{n}] \otimes \nabla \phi$ for homeotropic anchoring. The molecular field $\boldsymbol{h}$ arises from the free energies of the system [25],

$\boldsymbol{h}=K\left[-\nabla \cdot\left(\frac{1+\phi}{2} \nabla \boldsymbol{n}\right)+\frac{1+\phi}{2} \frac{\left(\boldsymbol{n}^{2}-1\right) \boldsymbol{n}}{\delta^{2}}\right]+\boldsymbol{g}$,

with $\boldsymbol{g}=A(\boldsymbol{n} \cdot \nabla \phi) \nabla \phi$ for planar anchoring, and $\boldsymbol{g}=$ $A[(\nabla \phi \cdot \nabla \phi) \boldsymbol{n}-(\boldsymbol{n} \cdot \nabla \phi) \nabla \phi]$ for homeotropic anchoring. The parameters $\gamma_{1}$ and $\gamma_{2}$ determine the relaxation time of $\phi$ and $\boldsymbol{n}$, and $\mu$ is the Newtonian viscosity of both components. We have assumed equal densities and viscosities between the two components although the theoretical model allows mismatched material properties [24].

To arrive at the Cahn-Hilliard equation (7) and the molecular field in (10), we have omitted from the right-hand side "coupling terms" between $f_{\text {mix }}$ and the nematic energies. These are insignificant as long as the interface stays narrow. In fact, the Cahn-Hilliard diffusive dynamics has a visible effect only during singular events such as film rupture [27]. In the current context, the diffuse interface may be seen as merely a numerical device for treating a moving internal boundary. In Eqs. (8) and (9), we have omitted respectively the viscous torque due to straining flow and anisotropic viscosities. Thus, we are using a simplified version of the Leslie-Ericksen theory [25]. These simplifications reflect a trade-off between capturing the complete physics and numerical convenience [16].

We use a two-dimensional (2D) Fourier spectral method for solving these equations, and an interested reader can find details of the numerical algorithm in Refs. [16,24,28]. All simulations are done on a regular mesh in a rectangular domain with periodic boundary conditions in both directions. To enhance stability, we advance time semi-implicitly, with the nonlinear transport terms treated explicitly while the linear terms implicitly. The algorithm is highly efficient; the number of operations per time step scales as $O(N \log N)$, $N$ being the number of unknowns. We have carried out mesh and time-step refinements to determine the adequacy of our spatial and temporal resolutions. The interface consists in a layer of thickness on the order of $5 \epsilon$ across which $\phi$ varies steeply. This layer typically requires $7-10$ grid points to resolve; fewer points would produce spurious oscillations in the solution [16]. The total number of grid points is typically $1024 \times 1024$. For the time step, we find that the temporal resolution is adequate as long as the simulation is stable, and that the Courant-Friedricks-Lewy condition is a good guideline for the stability of our semi-implicit scheme.

\section{Results and discussion}

The geometry of the problem is shown in Fig. 1. An elliptic nematic drop is suspended in a Newtonian fluid, its semi-axes being $L_{0}$ and $B_{0}$ initially. There is zero velocity and zero stress throughout the domain. Then the drop begins to retract under the action of interfacial tension. This roughly represents the drop retraction experiments that start by melting a short LCP fiber sandwiched between layers of a flexible polymer (e.g., [20]). We assume that the fluids are highly viscous and the retraction is slow so that inertia is negligible. This is justified for the typical materials used in experiments.

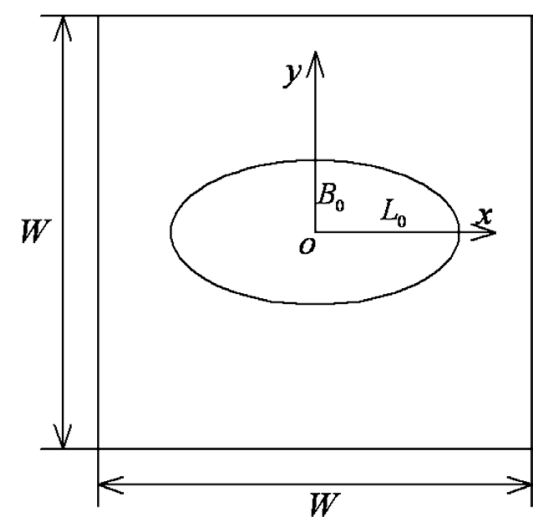

Fig. 1. Computational domain for drop retraction. 
We have simulated three cases with the drop being Newtonian (baseline case), nematic with planar anchoring and nematic with homeotropic anchoring. We will use the final drop radius $R_{0}=\sqrt{L_{0} B_{0}}$ as the characteristic length. The dimensionless geometric parameters are $W=3.9738$, $L_{0}=1.5811$ and $B_{0}=0.6325$. Using the capillary time scale $\mu R_{0} / \sigma$, we make the model parameters dimensionless: $\lambda=1.342 \times 10^{-2}, \gamma_{1}=4 \times 10^{-5}, \epsilon=1.265 \times 10^{-2}$, $\delta=6.325 \times 10^{-2}, K=6.708 \times 10^{-2}, A=6.708 \times 10^{-3}$ and $\gamma_{2}=10$. Compared with the material constants of common liquid crystals and liquid crystal polymers [29], the anchoring energy $A$ is in the realistic range, while the bulk elastic constant $K$ and director relaxation parameter $\gamma_{2}$ are about an order of magnitude too large. These are used to amplify the novel effects brought on by the liquid crystallinity inside the drop. For planar anchoring, $\boldsymbol{n}$ is initially horizontal everywhere. For homeotropic anchoring, we impose a radial $\boldsymbol{n}$ emanating from the center of the drop. Since the drop is elliptic, the initial $\boldsymbol{n}$ field deviates from the easy direction over much of the interface in both cases. The calculations are in $2 \mathrm{D}$, but we expect the physical insights gained here to be relevant both to the sharp-interface theory [13] and to 3D experiments $[19,20]$.

\subsection{Drop retraction and apparent interfacial tension}

Fig. 2 shows that the nematic drop with homeotropic anchoring retracts faster than the Newtonian drop while the one with planar anchoring is slower than the Newtonian drop. Heuristically, these results can be explained in terms of the anchoring energy $f_{\text {anch }}$ [16]. Given the initial $\boldsymbol{n}$ fields, the retraction reduces $f_{\text {anch }}$ for homeotropic anchoring but increases it for planar anchoring. The final equilibrium shape is circular for homeotropic anchoring, with a hedgehog defect at the center. For planar anchoring, the final shape is prolate. The director field exhibits the well-known bipolar configuration with two surface defects at the poles $[2,30]$. If we use smaller and more realistic $K$ values, the bipolar drop will be nearly circular. Yue et al. [16] have showed a plot

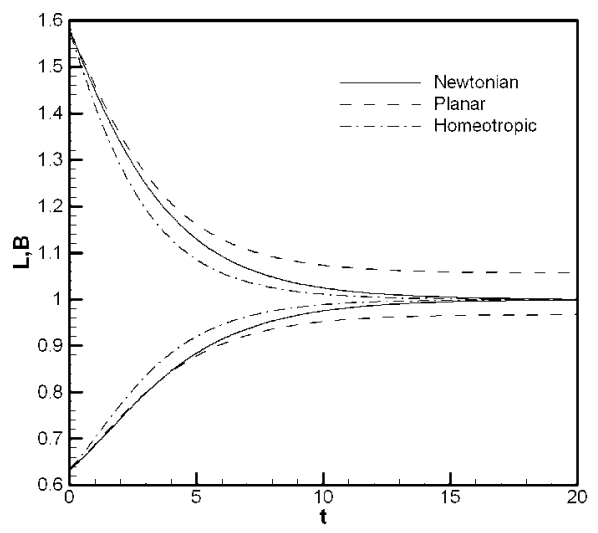

Fig. 2. Drop retraction: variation of the major and minor semi-axes for a Newtonian drop and nematic drops with planar and homeotropic anchoring (after Ref. [16]; C Cambridge Univ. Press). similar to Fig. 2 to illustrate the capability of the method. Here we review the solutions as an introduction to the main results in the next two subsections.

As mentioned in Section 1, drop retraction is a convenient method for measuring the interfacial tension between the drop and matrix fluids $[17,18]$. By assuming an ellipsoidal drop shape and Newtonian rheology in both components, Maffettone and Minale [31] developed a phenomenological relationship between the shape relaxation of the drop and the interfacial tension,

$$
\begin{aligned}
L^{2}-B^{2}= & \left(L^{2}-B^{2}\right)_{t=0} \\
& \times \exp \left[-\frac{\sigma t}{\mu_{m} R_{0}} \frac{40(\beta+1)}{(2 \beta+3)(19 \beta+16)}\right],
\end{aligned}
$$

where $R_{0}$ is the equilibrium drop radius, $\mu_{m}$ is the matrix viscosity and $\beta$ is the viscosity ratio between the drop and the matrix. By measuring $L(t)$ and $B(t)$, the half-length and half-width of the drop, the interfacial tension $\sigma$ can be calculated from curve fitting. The retraction curves for nematic drops in Fig. 2 do not obey the exponential scaling of Eq. (11). However, if we use the local slope of the curves to fit the formula, we may determine an apparent interfacial tension $\sigma_{\text {app }}$ at different times. This is plotted in Fig. 3 for the three cases. The initial increase in $\sigma_{\text {app }}$ is a numerical artifact due to the relaxation of the imposed initial $\phi$ profile [16]. Afterward, $\sigma_{\text {app }}$ stays constant for the Newtonian drop, as expected. For the drop with homeotropic anchoring, $\sigma_{\text {app }}$ is larger on account of the faster retraction; it is also roughly constant until the end of the retraction. In contrast, the drop with planar anchoring has a lower $\sigma_{\text {app }}$ and it declines in time.

Experimentally, Yu et al. [20] found that the retraction of a thermotropic LCP drop deviates from the exponential law in Eq. (11). They have determined $\sigma_{\text {app }}$ using the local slope of the retraction curves, and found that it indeed declines in time. Therefore, one may use the heuristic energy argument to explain the experiment if the anchoring is planar in the experimental drops. Unfortunately, this could not be determined for the thermotropic LCP drops in Ref. [20]. Visualizations on a drop of a different LCP indicate that this is indeed the case [32]. In addition, the interior of the drop exhibits the well-known polydomain texture, with little orientational order as a whole [29]. This explains why the experimental drops retract completely to a spherical shape while our drop with planar anchoring does not. In the experiment of $\mathrm{Yu}$ et al. [20], the declining $\sigma_{\mathrm{app}}$ is probably a combined effect of $f_{\text {anch }}$ and the relaxation of the microdomains. Using still a different pair of polymers, Lee and Denn [19] reported faster retraction for smaller LCP drops in terms of a dimensionless retraction time scaled by the capillary time. The authors argued that this reflects the effect of distortional elasticity, which is prominent for drops with sizes comparable to the domain size. This would be consistent with our results if the anchoring in the experimental drop is homeotropic. But no information is given on the anchoring in Ref. [19]. 


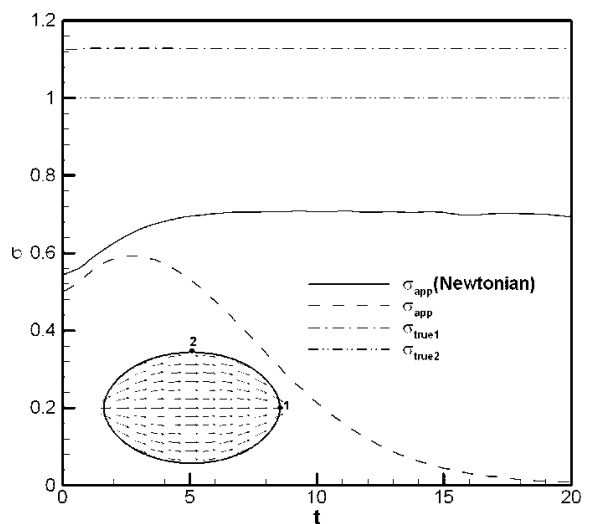

(a)

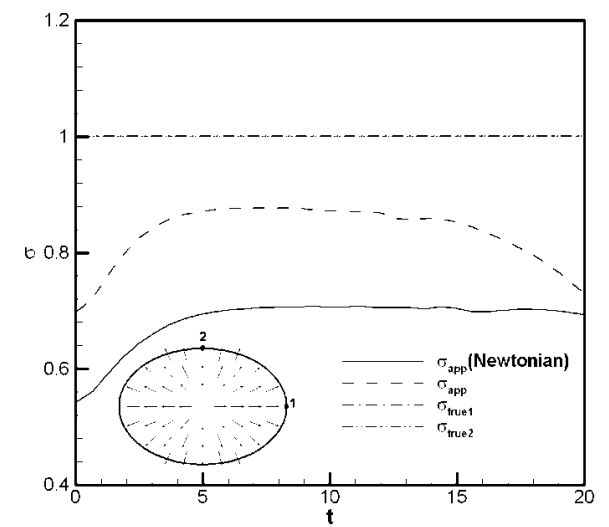

(b)

Fig. 3. The apparent interfacial tension $\sigma_{\text {app }}$ compared with the true interfacial tension $\sigma$ calculated from the interfacial energy (Eq. (12)). (a) Bipolar configuration; (b) homeotropic configuration. The apparent interfacial tension for the Newtonian drop is also shown for comparison.

To sum up this subsection, our simulations show that the retraction of nematic drops differs from that of Newtonian drops. In terms of an apparent interfacial tension $\sigma_{\text {app }}$, our results seem to explain the experimental observations. However, $\sigma_{\text {app }}$ obviously does not represent the true interfacial forces during retraction.

\subsection{True dynamic interfacial tension and Marangoni flow}

The true dynamic interfacial tension $\sigma_{\text {true }}$, defined as the interfacial energy density, varies with time as well as location along the interface. Because our theoretical model is based on the free energy, it is very convenient to calculate $\sigma_{\text {true }}$ from the equivalent of the interfacial energy per unit area in the diffuse-interface representation,

$\sigma_{\text {true }}=\int\left(f_{\text {mix }}+f_{\text {anch }}\right) \mathrm{d} h$,

where the integration is performed across the interface. As $f_{\text {anch }}$ may vary along the interface because of different anchoring angles, so may the true dynamic interfacial tension $\sigma_{\text {true }}$. We plot the true interfacial tension at the tip (Point 1) and waist (Point 2) of the drop in Fig. 3. If the director $\boldsymbol{n}$ is oriented along the easy direction everywhere on the interface, $f_{\text {anch }}$ vanishes. Then $\sigma_{\text {true }}$ reduces to the usual interfacial tension due to $f_{\text {mix }}$, which is roughly a constant and taken to be unity here because of our scaling. For planar anchoring, the director $\boldsymbol{n}$ is almost perpendicular to the interface at Point 1, thus $f_{\text {anch }}>0$ there and the true interfacial tension $\sigma_{\text {true } 1}$ is greater than unity. At Point 2 the true interfacial tension $\sigma_{\text {true } 2}$ stays at unity because $\boldsymbol{n}$ is always parallel to the interface at that point, which produces zero anchoring energy (cf. Eq. (3)). This clearly illustrates how the bulk orientation field influences the interfacial tension. By the same token, the homeotropic anchoring is satisfied at both points in Fig. $3 \mathrm{~b}$, and $\sigma_{\text {true } 1}$ and $\sigma_{\text {true } 2}$ both remain roughly constant at unity. The lack of temporal variation of $\sigma$ in Fig. 3 is coincidental because our initial conditions are such that $f_{\text {anch }}$ barely changes at the two points during the retraction. For points between 1 and $2, \sigma$ should vary in time. Note that the apparent interfacial tension is always below unity: $\sigma_{\mathrm{app}}<1$, even for the Newtonian drop. This is because the Maffettone-Minale formula is intended for three dimensions, and our two-dimensional drop retracts more slowly.

When the anchoring energy varies along the interface, the gradient of the dynamic interfacial tension drives a Marangoni flow. Fig. 4a shows the velocity field near the nematic drop with planar anchoring toward the end of the retraction. The flow goes from Point 2 to Point 1, along the gradient of the interfacial tension. For the radial configuration in Fig. 4b, the Marangoni flow is negligible for lack of a tangential gradient in $\sigma_{\text {true }}$. As far as we know, there has been no report of experimental observation of such nematic Marangoni flows. In reality, we expect the Marangoni effect to be less prominent because our elastic constant $K$ is intentionally too large. For a smaller $K$, the interior of the $\boldsymbol{n}$ field will be adapted more readily to satisfy the anchoring condition and $f_{\text {anch }}$ will be reduced. With strong anchoring, a bipolar drop tends to develop cusp-like protrusions at the poles in response to the surface defects [33]. The director thus deviates from the easy axis only in a very small region at the tip. In addition, the large curvature in the region will generate a high pressure; the pressure gradient along the interface may act to reduce the Marangoni flow.

As mentioned in Section 1, our theoretical model is a diffuse-interface implementation of the ideas that Rey put forth [13], and our numerical results bear out the main predictions of Rey's sharp-interface theory. The first and rather obvious observation is that the interfacial tension on a nematic-isotropic interface depends on the director orientation in the bulk. This has been shown by the discrepancy between $\sigma_{\text {true } 1}$ and $\sigma_{\text {true } 2}$ in Fig. 3a and the lack thereof in Fig. 3b. This picture is also consistent with the Monte Carlo simulations of $\mathrm{Li}$ and Denn [10] showing the effect of far-field orientation on the interface tension, although the form of the anchoring energy is postulated here (Eq. (3)) but is the result of chain interactions in Ref. [10]. Secondly, the tangential gradient of the interfacial tension generates a Marangoni flow from regions of lower anchoring energy to regions of higher energy, as anticipated by Rey's sharp- 


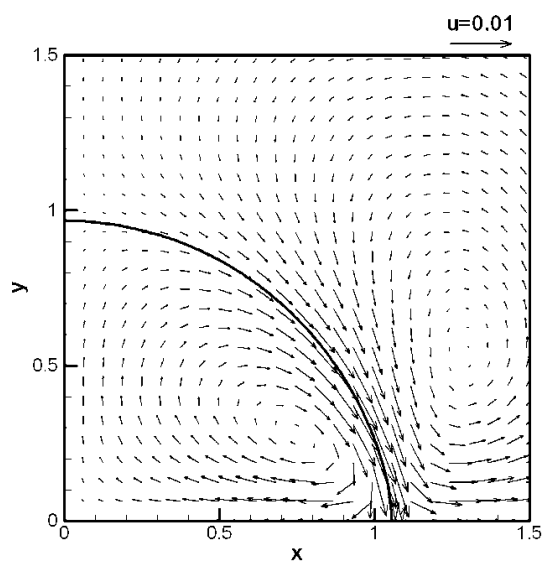

(a)

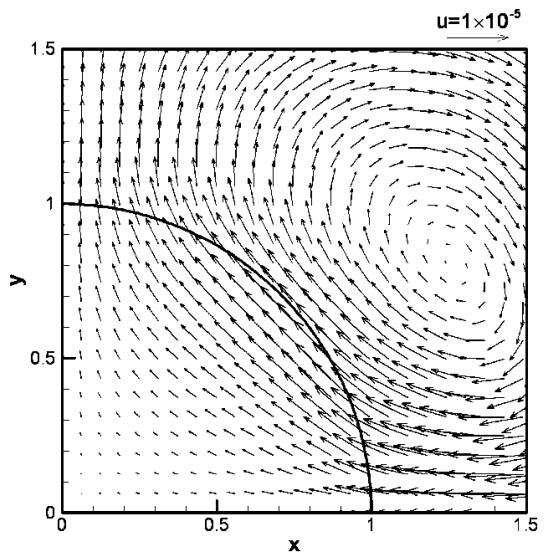

(b)

Fig. 4. Velocity field at $t=29.82$ near the nematic drop. (a) Bipolar configuration, where a Marangoni flow prevails. (b) Homeotropic configuration, where the velocity is negligible compared with that in (a).

interface theory $[11,12]$. The idea of the anchoring energy producing a bending stress will be discussed next.

\subsection{Normal force and effective interfacial tension}

According to the true interfacial tension in Fig. 3, the nematic drop with planar anchoring should retract faster than the Newtonian drop owing to the larger dynamic interfacial tension $\sigma$, while that with homeotropic anchoring should retract with roughly the same speed as the Newtonian drop. This expectation is at odds with Fig. 2. The reason is that besides the interfacial tension, the anchoring energy generates a normal surface force that influences the retraction as well. To illustrate this mechanism, we will use the planar anchoring case as an example to analyze the interfacial forces.

Fig. 5 shows a pillbox control-volume that covers the interface. Yue et al. [16] have derived the stress tensors due to the mixing energy $f_{\text {mix }}$ and the anchoring energy $f_{\text {anch }}$ (see also Eq. (9)):

$\boldsymbol{T}_{\text {mix }}=f_{\text {mix }} \boldsymbol{I}-\lambda(\nabla \phi \otimes \nabla \phi)$,

$\boldsymbol{T}_{\text {anch }}=f_{\text {anch }} \boldsymbol{I}-A(\boldsymbol{n} \cdot \nabla \phi) \boldsymbol{n} \otimes \nabla \phi$.

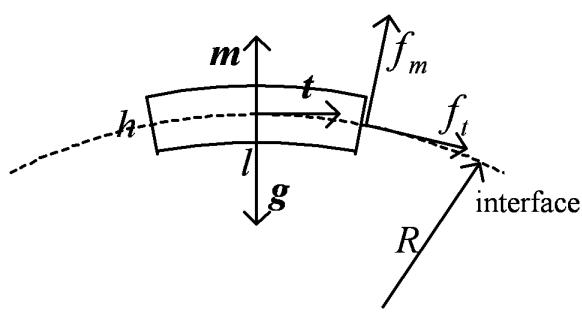

Fig. 5. A pillbox control-volume, of thickness $h$ and width $l$, for calculating interfacial forces. $R$ is the radius of curvature of the interface and $\boldsymbol{m}$ and $\boldsymbol{t}$ are unit normal and tangential vectors. See text for the definition of the surface forces $\boldsymbol{g}, f_{t}$ and $f_{m}$.

From the total stress tensor $\boldsymbol{T}=\boldsymbol{T}_{\text {mix }}+\boldsymbol{T}_{\text {anch }}$, we can calculate the tangential traction $f_{t}$ and the normal traction $f_{m}$ on the sides of the pillbox. Noting that $\nabla \phi$ is parallel to the surface normal $\boldsymbol{m}$ and perpendicular to $\boldsymbol{t}$, we obtain

$$
\begin{aligned}
f_{t} & =\int(\boldsymbol{t} \cdot \boldsymbol{T} \cdot \boldsymbol{t}) \mathrm{d} h=\int\left(f_{\text {mix }}+f_{\text {anch }}\right) \mathrm{d} h, \\
f_{m} & =\int(\boldsymbol{t} \cdot \boldsymbol{T} \cdot \boldsymbol{m}) \mathrm{d} h \\
& =-\int A(\boldsymbol{n} \cdot \nabla \phi)(\boldsymbol{t} \cdot \boldsymbol{n})(\nabla \phi \cdot \boldsymbol{m}) \mathrm{d} h,
\end{aligned}
$$

where the integration is performed across the interface. Now $f_{t}$ is the dynamic interfacial tension in Eq. (12). But $f_{m}$ is an additional normal force that stems from the anchoring energy. Although this force does not stretch the interface, it nevertheless affects the retraction of the drop in a way analyzed below.

The total force per unit area due to the interfacial energy is $\boldsymbol{g}=\int(\nabla \cdot \boldsymbol{T}) \mathrm{d} h$, and $g=\boldsymbol{g} \cdot(-\boldsymbol{m})$ is the normal traction pushing the drop inward. In view of the Young-Laplace equation, we may consider $g R$ a local "effective interfacial tension" that determines the retraction of the drop, $R$ being the local radius of curvature. Fig. 6a plots $g R$ at the tip and waist of the drop with planar anchoring. Our scaling is such that for a Newtonian drop, the effective interfacial tension $g R=1$. The initial transient $(t \leqslant 1)$ is a numerical artifact due to the relaxation of the initially imposed $\phi$ profile and $\boldsymbol{n}$ field that do not happen to minimize the system's free energy for the initial drop shape [16].

For the bipolar configuration, $(g R)_{1}<1<(g R)_{2}$. This implies that compared with a Newtonian drop, the normal force pushing inward is stronger at the waist and weaker at the pointed ends. As a result, the nematic drop retracts more slowly than the Newtonian drop as seen in Fig. 2. Thus, the apparent interfacial tension $\sigma_{\text {app }}$ estimated from the retraction curves is smaller than that of the Newtonian drop (Fig. 3a). The further divergence of $(g R)_{1}$ and $(g R)_{2}$ in time causes the rate of retraction, and hence $\sigma_{\text {app }}$, to decrease with time. The later fall of $\sigma_{\text {app }}$ toward zero is because the bipolar drop fails to attain a circular shape.

A similar analysis of the surface forces can be carried out for the homeotropically anchored drop (Fig. 6b), although the expression for the normal force will differ from Eq. (16). For $t \leqslant 12,(g R)_{1}>1>(g R)_{2}$, implying that the anchoring 


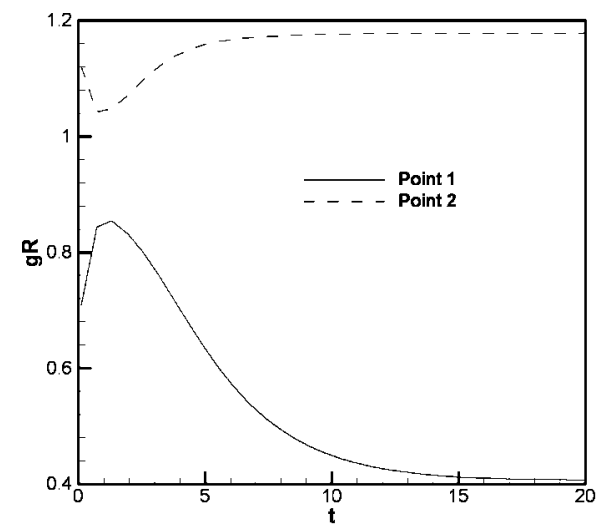

(a)

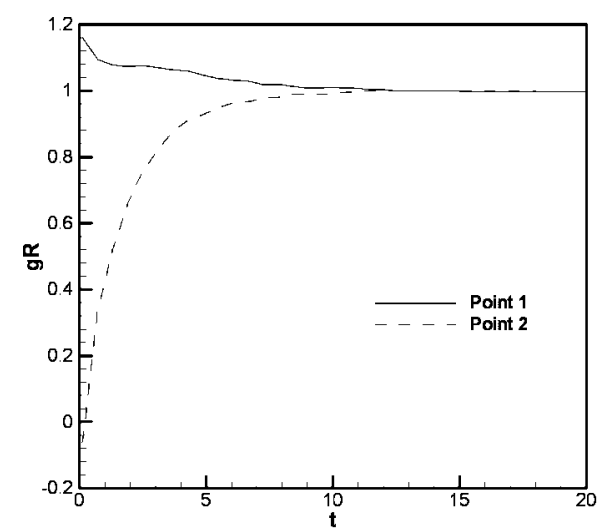

(b)

Fig. 6. The effective interfacial tension $g R$ at the waist (Point 1 ) and pole (Point 2) of the drop, where $g$ is the total normal traction pushing inward, and $R$ is the local radius of curvature calculated by assuming an elliptic drop. (a) Planar anchoring; (b) homeotropic anchoring.

energy will make the retraction go faster than for the Newtonian drop. This explains Fig. 2 as well as the higher $\sigma_{\text {app }}$ in Fig. 3b. For $t>13,(g R)_{2}$ exceeds $(g R)_{1}$ slightly. The retraction of the homeotropic drop is thus hampered toward the end, consistent with the fall of $\sigma_{\text {app }}$ in Fig. $3 \mathrm{~b}$.

We conclude this section by noting that the normal force $f_{m}$ corresponds to the bending stress in the sharp-interface theory of Rey [13]. In fact, our expression in Eq. (16) is precisely the diffuse-interface version of the bending stress in Ref. [13] (Eqs. (21)-(23) therein). Our simulation of drop retraction has confirmed the role of this normal stress in moderating the motion of nematic-isotropic interfaces.

\section{Concluding remarks}

In this paper, we have used a diffuse-interface formulation to simulate the retraction of a liquid-crystalline drop in an isotropic fluid. The anchoring energy $f_{\text {anch }}$ plays a fundamental role in the interfacial dynamics of nematic liquids. In particular, it gives rise to a dynamic interfacial tension that may vary along the interface. The spatial gradient of the interfacial tension drives a Marangoni flow near the nematicisotropic interface. Finally, the anchoring energy produces an additional normal force on the interface that, together with the interfacial tension, determines the retraction of the drop.

A major objective of this work is to demonstrate the novel interfacial dynamics on a nematic-isotropic interface in a nontrivial flow. This has been accomplished; the three effects of $f_{\text {anch }}$ listed above clearly substantiate the theoretical expectations given in Section 1. Unfortunately, we know of no experimental study that probes these effects, and can only seek indirect corroboration in drop retraction experiments. The numerical results seem to be consistent with the few experimental observations, subject to the restriction that we have not accounted for the polydomain texture. Because of $f_{\text {anch, }}$ one cannot define an interfacial tension as a material constant between a nematic and an isotropic liquid, much less measure it from the shape evolution during drop retraction.

We have to point out several limitations in our work. Recall that our nematic is represented by a simplified LeslieEricksen theory. We have adopted a single elastic constant and a single viscosity (cf. Eq. (9)). Real nematics, smallmolecule and polymeric ones alike, exhibit anisotropic elasticity and viscosities that would introduce additional complications in the retraction of the nematic drop. Even the full Leslie-Ericksen theory fails to represent polymeric nematics and defect-laden textures [29]. In addition, the parameter values are chosen not to represent certain real liquid crystals but to highlight the novel physical effects. Furthermore, the simulation is in two dimensions, where the retraction occurs more slowly than in three dimensions as noted in the discussions. As a consequence of these simplifications, the numerical results cannot quantitatively predict experiments. Rather, we expect the physical principles uncovered here to carry over qualitatively to reality. Work is under way to incorporate the full Leslie-Ericksen theory and simulate drop retraction in an axisymmetric geometry.

\section{Acknowledgments}

Acknowledgment is made to the Donors of the Petroleum Research Fund, administered by the American Chemical Society, for partial support of this research. J.J.F. was also supported by the NSF (CTS-0229298, CTS-9984402), the NSERC's Canada Research Chair and Discovery programs, and the NNSF of China (Nos. 20174024 and 20490220). J.S. was supported by the NSF (DMS-0074283, DMS-0311915). C.L. was supported by the NSF (DMS-0405850).

\section{References}

[1] F.P. La Mantia, Thermotropic Liquid Crystal Polymer Blends, Technomic, Lancaster, PA, 1993.

[2] J.L. West, in: R.A. Weiss, C.K. Ober (Eds.), Liquid-Crystalline Polymers, in: ACS Symp. Ser., vol. 435, ACS, Washington, DC, 1990, chap. 32, pp. 475-495. 
[3] A.A. Collyer, in: D. Acierno, A.A. Collyer (Eds.), Rheology and Processing of Liquid Crystal Polymers, Chapman \& Hall, New York, 1996, chap. 6, pp. 185-217.

[4] A.I. Isayev, in: A.I. Isayev, T. Kyu, S.Z.D. Cheng (Eds.), Liquid-Crystalline Polymer Systems: Technological Advances, in: ACS Symp. Ser., vol. 632, ACS, Washington, DC, 1996, chap. 1, pp. 1-20.

[5] M. Mucha, Prog. Polym. Sci. 28 (2003) 837-873.

[6] A. Rapini, M. Popoular, J. Phys. (Paris) C 30 (1969) 54-56.

[7] B. Jerome, Rep. Prog. Phys. 54 (1991) 391-451.

[8] Z.Y. Chen, J. Noolandi, Phys. Rev. A 45 (1992) 2389-2392.

[9] T.P. Doerr, P.L. Taylor, Mol. Cryst. Liq. Cryst. 330 (1999) 491-501.

[10] X. Li, M.M. Denn, Macromolecules 35 (2002) 6446-6454.

[11] A.D. Rey, J. Chem. Phys. 110 (1999) 9769-9770.

[12] A.D. Rey, Liq. Cryst. 26 (1999) 913-917.

[13] A.D. Rey, Phys. Rev. E 61 (2000) 1540-1549.

[14] A.D. Rey, J. Non-Newtonian Fluid Mech. 96 (2001) 45-62.

[15] V. Popa-Nita, T.J. Sluckin, Phys. Rev. E 66 (2002) 041703.

[16] P. Yue, J.J. Feng, C. Liu, J. Shen, J. Fluid Mech. 515 (2004) 293-317.

[17] H. Mo, C. Zhou, W. Yu, J. Non-Newtonian Fluid Mech. 91 (2000) 221-232.

[18] Y. Son, K.B. Migler, Polymer 43 (2002) 3001-3006.

[19] H.S. Lee, M.M. Denn, J. Non-Newtonian Fluid Mech. 93 (2000) 315323.
[20] R. Yu, W. Yu, C. Zhou, J.J. Feng, J. Appl. Polym. Sci. 94 (2004) 1404 1410.

[21] C. Liu, J. Shen, J.J. Feng, P. Yue, in: A. Miranville (Ed.), Mathematical Models and Methods in Phase Transitions, Nova Publications, 2005.

[22] J. Lowengrub, L. Truskinovsky, Proc. R. Soc. London A 454 (1998) $2617-2654$.

[23] D. Jacqmin, J. Comput. Phys. 155 (1999) 96-127.

[24] C. Liu, J. Shen, Physica D 179 (2003) 211-228.

[25] P.G. de Gennes, J. Prost, The Physics of Liquid Crystals, Oxford Univ. Press, New York, 1993.

[26] C. Liu, N.J. Walkington, SIAM J. Numer. Anal. 37 (2000) 725-741.

[27] P. Yue, J.J. Feng, C. Liu, J. Shen, J. Non-Newtonian Fluid Mech. (2005), in press.

[28] J. Shen, SIAM J. Sci. Comput. 16 (1995) 74-87.

[29] R.G. Larson, The Structure and Rheology of Complex Fluids, Oxford Univ. Press, New York, 1999.

[30] Q. Shen, C. Liu, M.C. Calderer, Contin. Mech. Thermodyn. 14 (2002) 363-375.

[31] P.L. Maffettone, M. Minale, J. Non-Newtonian Fluid Mech. 78 (1998) 227-241.

[32] J. Wu, P.T. Mather, in: Society of Rheology 74th Annual Meeting, Minneapolis, MN, 2002, Paper HS12.

[33] P. Yue, J.J. Feng, C. Liu, J. Shen, in preparation. 